HNO 2014 • 62:780-780

DOI 10.1007/s00106-014-2928-z

Online publiziert: 16. Oktober 2014

๑) Springer-Verlag Berlin Heidelberg 2014

\section{R. Knecht}

Klinik und Poliklinik für Hals-, Nasen und Ohrenheilkunde, Kopf-Hals-Chirurgie und Onkologie, Universitätsklinikum Hamburg-Eppendorf

\section{Aktuelle Therapiekonzepte bei Kopf-Hals-Tumoren}

\section{Highlights zum 50. Jubiläum der ASCO 2014}

In diesem Jahr fand das 50. Jubiläum der American Society of Clinical Oncology (ASCO) in Chicago statt. Auch dieses Jahr gab der Kongress einen guten Überblick über Status und Trends der KopfHals-Onkologie. Insgesamt besuchten knapp 35.000 Teilnehmer diesen internationalen Kongress, und Deutschland zählte zu den Top 3 der teilnehmenden Länder. Eine wachsende Anzahl von Studien weltweit macht die Wichtigkeit einer $\mathrm{Zu}$ sammenarbeit der verschiedenen Disziplinen deutlich. Längst ist die Therapie von Kopf-Hals-Tumoren nicht mehr nur in der Hand von HNO- oder MKGFachärzten. Multimodalität und Interdisziplinarität stehen bei einer aktuellen und zeitgemäßen Therapie von Kopf-Hals-Tumoren im Vordergrund, insbesondere bei den fortgeschrittenen Stadien. Eine onkologische Therapie nach zeitgemäßen und immer aktuellen Standards kann nur noch durch eine hohe Spezialisierung der einzelnen Fachexperten erreicht werden. Gleichzeitig ist es für eine optimale $\mathrm{Pa}$ tientenbehandlung notwendig, nicht nur chirurgische oder nichtchirurgische Behandlungsoptionen anzubieten, sondern auch die Vor- und Nachteile dieser Therapien genau zu kennen und auf den $\mathrm{Pa}$ tienten abzustimmen.

In den folgenden Übersichtsartikeln werden die wichtigsten Studienergebnisse zur Behandlung der Kopf-Hals-Karzinome zusammengefasst. Die bedeutendsten Neuigkeiten werden für die tägliche Praxis erfasst und dabei in den Kontext der Studienresultate der vergangenen Jahre eingeordnet. Diese werden in zwei separaten Artikeln vorgestellt, die sich zum einen mit der Primärtherapie und zum anderen mit der Rezidiv- und Metastasen-Situation befassen.

Im ersten Artikel geht es um aktuelle Studienergebnisse im Bereich der primären Radiochemotherapie, konkomitant und sequenziell. Hierbei sind in diesem Jahr wieder sehr interessante Resultate von Phase-III-Studien veröffentlicht worden. Nachdem vor 2 Jahren die ersten Ergebnisse von vergleichenden Studien zu konkomitanter und sequenzieller Radiochemotherapie eher enttäuschend waren, zeigen dieses Jahr zwei Induktionschemotherapie-Studien erfolgversprechende Ergebnisse. Im Rahmen von Organerhalt informierte eine Deutsche Studie über Ergebnisse einer Induktionschemotherapie in Kombination mit Cetuximab. Der EGFR-Antikörper wurde auch im Rahmen einer Studie zu HPV-assoziierten Oropharynxkarzinomen angewendet.

Im zweiten Artikel werden die wichtigsten Highlights im Bereich der Rezidiv- und Metastasenbehandlung dargestellt. Gerade in dieser Therapiesituation werden ständig zahlreiche Studien mit neuen, erfolgversprechenden Substanzen durchgeführt. Auch dieses Jahr gab es zahlreiche Studien, die neue Therapeutika als Optionen bei Therapieversagen von platinbasierten Regimes oder bei Versagen von sich daran anschließender Cetuximab-Erhaltungstherapie untersuchten. Doch die Langzeitdaten von der EXTREME-Studie machten erneut deutlich, dass trotz neuer Medikamente die Überlebensrate und die Prognose dieser Patienten weiterhin extrem schlecht sind.

Bemerkenswert ist, dass von 157 Beiträgen zu Kopf-Hals-Tumoren nur 4 Stu- dien in Kombination mit onkologischer Chirurgie durchgeführt wurden. Die Chirurgie wird auch in Zukunft trotz moderner Bestrahlungstechniken und innovativen Medikamenten immer eine Rolle in der Primärtherapie, Salvage- und Palliativsituation spielen. Umso wichtiger ist es, in Zukunft auch vermehrt chirurgische Therapieansätze mit in die interdisziplinären Studien einzubinden.

Wir hoffen, Ihnen hiermit eine Übersicht in der sich ständig wandelnden Forschungslandschaft im Bereich der KopfHals-Onkologie geben zu können.

Herzlichst

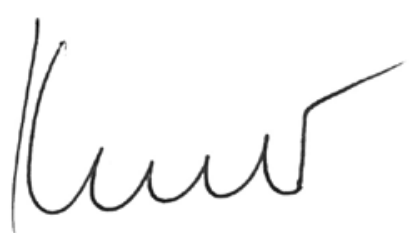

Ihr R. Knecht

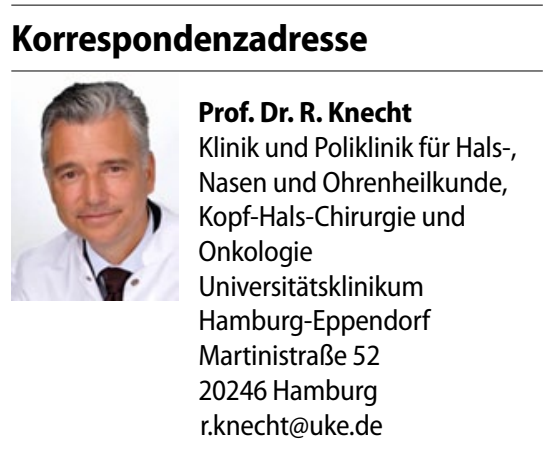

Interessenkonflikt. R. Knecht gibt an, dass kein Interessenkonflikt besteht. 